Conclusion: The immunogenicity of patients treated with RTX is a rare event with possible clinical and biological consequences, especially in patients with high antibody levels.

REFERENCES

[1] Smolen et al, Ann Rheum Dis 2017

[2] Tony et al, Arthritis Care Res 2019

Disclosure of Interests: None declared

DOI: 10.1136/annrheumdis-2021-eular.954

\section{POS0601 DIFFERENCES IN DRUG SURVIVAL BETWEEN ORIGINATOR AND BIOSIMILAR PRODUCTS AMONG FIRST USERS OF EACH MOLECULE}

D. Di Giuseppe ${ }^{1}$, H. Bower ${ }^{1}$, B. Delcoigne ${ }^{1}$, T. Frisell ${ }^{1}$, K. Chatzidionysiou ${ }^{1,2}$, U. Lindström ${ }^{3}$, C. Sjowall ${ }^{4}$, E. Lindqvist ${ }^{5}$, J. Askling ${ }^{1}$ on behalf of ARTIS. ${ }^{1}$ Karolinska Institute, Department of Medicine Solna, Stockholm, Sweden; ${ }^{2} \mathrm{Nya}$ Karolinska, Rheumatology Unit, Stockholm, Sweden; ${ }^{3}$ Sahlgrenska Academy, University of Gothenburg, Rheumatology Unit, Karolinska University Hospital, Gothenburg, Sweden; ${ }^{4}$ Linköping University, Division of Inflammation and Infection, Department of Biomedical and Clinical Sciences, Linköping, Sweden; ${ }^{5}$ Lund University, Skåne University Hospital, Department of clinical sciences, Rheumatology, Lund, Sweden

Background: Biosimilar products of biological disease-modifying antirheumatic drugs (bDMARDs) entered the Swedish market in 2015, with regulatory approvals based on head to head trials of limited duration. Longer-term comparative drug survival, in clinical practice, remains less well documented.

Objectives: To compare survival on drug between biosimilars and their originator products among first starters of etanercept, infliximab, adalimumab and rituximab.

Methods: Data from the Swedish Rheumatology Quality register (SRQ) was used to identify and follow patients who started a first ever treatment with etanercept since April 2015 (originator=ETA, biosimilar= SB4), infliximab since March 2014 (originator=IFX,biosimilar= CT-P13), adalimumab since January 2018 (originator=ADA biosimilars=SB5, ABP501), or rituximab since January 2018 (originator=RIT, biosimilar= GP2013), through December $31^{\text {st }}, 2019$, date of first discontinuation of the drug, or death. Discontinuation was defined as lack of effectiveness or adverse events, while other reasons for interruption of the drug (including non-medical switch) were considered censoring events. Descriptive characteristics were collected from the SRQ and tabulated. Hazard ratios (HR) of discontinuation were estimated using Cox regression, with each drug analyzed separately, adjusted for age,sex, indication, line of treatment,disease duration,year of treatment start,region and concomitant use of csDMARD.

Results: 9274 patients started etanercept(49\% SB4), 3609 started infliximab(64\% CT-P13), 3117 started adalimumab(27\% SB5, 14\% ABP 501), and 763 started rituximab(39\% GP2013), Table 1. Patients starting CT-P13 and GP2013 were less likely to be biologics-naive compared to those starting the originator product. Initiators of SB5,ABP501 and GP2013 were more likely, and those starting CT-P13 were less likely,to be on concomitant csDMARDs compared to those starting the originator products. Patients characteristics of ETA and SB4 were similar.

The introduction of a biosimilar was typically followed by a decrease in the uptake of the originator, but for ETA a change in pricing in 2018 later led to a reversal of this pattern (Figure 1).

For IFX,ADA, and RIT, survival on drug was similar for the originator and its biosimilar(s). For ETA,risk of discontinuation was somewhat lower for the biosimilar than for the originator(adjusted HR:0.87,95\% confidence interval:0.79-0.95), Table 1.

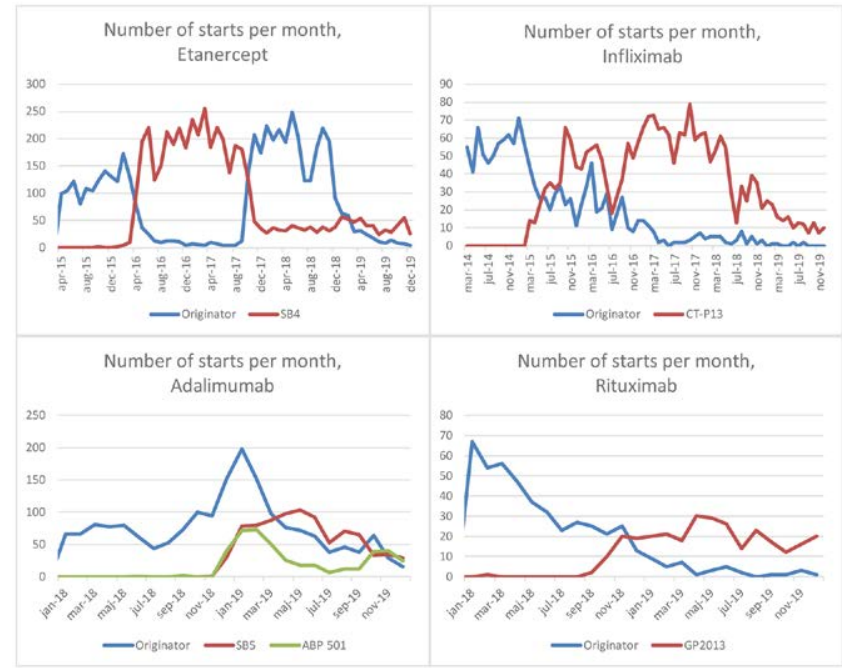

Figure 1. Number of starts of biosimilars compared to the originator during the follow-up time, by molecule

Conclusion: Despite their identical indications and therapeutic positioning, there are some differences in the baseline characteristics between patients who start ADA, IFX and RIT and their biosimilars. There are no differences in drug survival between originator and biosimilar with the possible exception of etanercept although the observed difference should be interpreted in light of possible unmeasured or residual channeling

Disclosure of Interests: Daniela Di Giuseppe: None declared, Hannah Bower: None declared, Bénédicte Delcoigne: None declared, Thomas Frisell: None declared, Katerina Chatzidionysiou Consultant of: Eli Lilly, AbbVie and Pfizer, Ulf Lindström: None declared, Christopher Sjowall: None declared, Elisabet Lindqvist: None declared, Johan Askling Grant/research support from: Abbvie, AstraZeneca, BMS, Eli Lilly, MSD, Pfizer, Roche, Samsung Bioepis, Sanofi, and UCB DOI: 10.1136/annrheumdis-2021-eular.977

\section{POS0602 ROLE OF INTRAARTICULAR BIOLOGICS AND METHOTREXATE IN THE MANAGEMENT OF ACTIVE INFLAMMATORY MONOARTHRITIS}

F. Aslam ${ }^{1}$, M. Meyer ${ }^{1}$, M. Pham ${ }^{2} .{ }^{1}$ Mayo Clinic, Rheumatology, Scottsdale, United States of America; ${ }^{2}$ Mayo Clinic, Medicine, Scottsdale, United States of America

Background: Expansion of immunosuppressive medications has greatly improved disease activity control in rheumatoid arthritis (RA) patients. However, persistent symptomatic monoarthritis can be a problem in some patients who otherwise have satisfactory disease control. Intra-articular (IA) corticosteroid (CS) injections have remained a treatment cornerstone for these patients, but the need for other therapies is clear. Patients can fail such therapy, may develop adverse effects or have comorbidities that are exacerbated by IA CS. $50 \%$ of patients relapse after IA CS therapy. Several studies have looked at other agents such as methotrexate (MTX) or tumor necrosis factor inhibitors (TNF-i) as IA treatment options.

Objectives: To systematically review the evidence regarding utility of IA methotrexate and biologics in managing inflammatory monoarthritis in patients with RA

Table 1. Hazard ratios of discontinuation and descriptive characteristics of biosimilar vs. originator among first starters of each molecule, until $31^{\text {st }}$ December 2019

\begin{tabular}{|c|c|c|c|c|c|c|c|c|c|}
\hline & \multicolumn{2}{|l|}{ Etanercept } & \multicolumn{2}{|l|}{ Infliximab } & \multicolumn{3}{|c|}{ Adalimumab } & \multicolumn{2}{|l|}{ Rituximab } \\
\hline & Originator & SB4 & Originator & CT-P13 & Originator & SB5 & ABP 501 & Originator & GP2013 \\
\hline $\mathrm{N}$ & 4721 & 4553 & 1308 & 2301 & 1834 & 852 & 431 & 465 & 298 \\
\hline Discontinuation & 1289 & 1236 & 582 & 878 & 399 & 139 & 80 & 57 & 26 \\
\hline Adjusted hazard ratios* & Ref & $0.87(0.79-0.95)$ & Ref & $1.14(0.99-1.31)$ & Ref & $1.02(0.83-1.26)$ & $1.16(0.88-1.52)$ & Ref & $1.12(0.68-1.85)$ \\
\hline Age, mean years $(\mathrm{std})$ & $51(16)$ & $51(15)$ & $49(16)$ & $49(16)$ & $48(15)$ & $52(15)$ & $51(15)$ & $59(15)$ & $60(15)$ \\
\hline Female, \% & $67 \%$ & $65 \%$ & $61 \%$ & $64 \%$ & $62 \%$ & $64 \%$ & $65 \%$ & $75 \%$ & $76 \%$ \\
\hline RA, \% & $46 \%$ & $48 \%$ & $39 \%$ & $35 \%$ & $33 \%$ & $42 \%$ & $43 \%$ & $61 \%$ & $76 \%$ \\
\hline Bionaïve, \% & $72 \%$ & $72 \%$ & $76 \%$ & $69 \%$ & $45 \%$ & $52 \%$ & $43 \%$ & $53 \%$ & $38 \%$ \\
\hline Disease duration, mean years (std) & $11(12)$ & $11(11)$ & $11(11)$ & $11(11)$ & $12(13)$ & $12(11)$ & $14(15)$ & $14(19)$ & $15(11)$ \\
\hline DAS28, mean & $4.0(1.3)$ & $4.0(1.4)$ & $4.1(1.4)$ & $4.1(1.4)$ & $3.7(1.4)$ & $3.8(1.3)$ & $4.0(1.3)$ & $4.5(1.4)$ & $4.7(1.4)$ \\
\hline Concomitant csDMARDs, \% & $45 \%$ & $47 \%$ & $57 \%$ & $48 \%$ & $37 \%$ & $49 \%$ & $42 \%$ & $36 \%$ & $43 \%$ \\
\hline
\end{tabular}

Abbreviations: RA=rheumatoid arthritis. csDMARDs=conventional synthetic DMARD, std=standard deviation. 
Methods: A systematic literature review, in accordance with PRISMA guidelines, was conducted looking at trials investigating the use of IA MTX and/or TNF-i, against IA CS, in managing inflammatory monoarthritis in patients with RA. A trained librarian conducted search of Ovid MEDLINE, Ovid EMBASE, Scopus, and Web of Science databases. Included studies were assessed for risk of bias as per the Cochrane tool.

Results: A total of 1013 citations were retrieved from the medical database searches. 12 studies were included in the final review. 6 studies investigated IA MTX while 5 studied IA etanercept. One study investigated different biologic agents: infliximab, etanercept, or adalimumab. Multitude of dosing regimens and administration protocols were used in various studies. 5 of the 6 MTX studies only included knees. IA MTX was compared against saline, CS and as combined MTX/CS vs CS alone. There was no evidence to support the use of IA MTX as a superior or equivalent agent to the comparator groups. Unlike MTX, etanercept was investigated in multiple joint types e.g. elbows, knees and wrists. IA etanercept was found to have utility as a successful IA alternative to CS. The only study investigating infliximab and adalimumab found them to be superior to IA CS across multiple joint types. All therapies were generally well tolerated. Etanercept studies had low risk of bias while the MTX studies mostly suffered from higher risk of bias.

Conclusion: Etanercept may be used as an IA agent in select patients with inflammatory arthritis when systemic treatment is not an option and IA corticosteroids cannot be used. Limited data also supports the utility of IA adalimumab and infliximab as better IA agents compared to CS. IA MTX, however, did not have such supporting data.

REFERENCES:

11] Fisher BA, Keat A. (2006) Should we be using intraarticular tumor necrosis factor blockade in inflammatory monoarthritis? J Rheumatol. 33(10), 1934-5.

[2] Weitoft T, Uddenfeldt P. (2000) Importance of synovial fluid aspiration when injecting intra-articular corticosteroids. Ann Rheum Dis. 59(3), 233-5.

Disclosure of Interests: None declared

DOI: 10.1136/annrheumdis-2021-eular.1016

\section{POS0603 ANALYSIS OF FACTORS ASSOCIATED WITH THE EFFECTIVENESS OF ABATACEPT IN THE ORIGAMI STUDY}

K. Misaki ${ }^{1}$, E. Tanaka ${ }^{2}$, E. Inoue ${ }^{3}$, K. Tsuritani $^{4}$, S. Matsumoto ${ }^{5}$, H. Yamanaka ${ }^{2,6}$, M. Harigai ${ }^{2}{ }^{1}$ Kita-Harima Medical Center, Rheumatology, Ono, Japan; ${ }^{2}$ Tokyo Women's Medical University School of Medicine, Department of Rheumatology, Tokyo, Japan; ${ }^{3}$ Showa University, Showa University Research Administration Center, Tokyo, Japan; ${ }^{4}$ Bristol-Myers Squibb K.K., Immunology Medical, Tokyo, Japan; ${ }^{5}$ Ono pharmaceutical Co, Ltd., Medical Affairs, Tokyo, Japan; ${ }^{6}$ Sanno Medical Center, Rheumatology, Tokyo, Japan

Background: The ORIGAMI study is a multicenter, observational study to evaluate the effectiveness, safety, and patient-reported outcomes of abatacept $(A B A)$ in Japanese patients with csDMARD-resistant, Simplified Disease Activity Index (SDAI)-moderate, biologic-naïve rheumatoid arthritis (RA). ABA has shown better effectiveness/efficacy in RA patients with anti-cyclic citrullinated peptide antibody (ACPA) positive (1) and high ACPA titer (2) compared to ACPA negative and Iow ACPA titer, respectively. However, more accurate predictors of effectiveness in clinical practice are needed than ACPA status.

Objectives: This post-hoc analysis is aimed to determine the association between ACPA and ABA effectiveness (disease activity and physical function) or retention rate and to investigate other factors associated with the effectiveness of ABA in patients enrolled in the ORIGAMI study.

Methods: Of the 279 patients in the effectiveness analysis set of the ORIGAMI study, 270 patients with baseline ACPA measurement were analyzed. The patients were divided into the ACPA-positive group (ACPA +ve, $\geq 4.5 \mathrm{U} / \mathrm{mL}$ at baseline) and the ACPA-negative group (ACPA -ve, $<4.5 \mathrm{U} / \mathrm{mL}$ ). Patients' characteristics, changes in disease activity and physical function (Japanese Health Assessment Questionnaire; J-HAQ) through 52 weeks, and retention rates of $\mathrm{ABA}$ at week 52 were evaluated. Baseline characteristics and use of concomitant drugs were analyzed as independent variables by multiple regression analysis using a standard linear model adjusted by SDAI at week 0 to identify factors associated with SDAI change at week 52 . In addition, the interaction effects among ACPA status, RF status, and the factor that was significantly associated with SDAI change in multiple regression analysis on changes in SDAI were explored.

Results: The numbers of ACPA +ve and -ve patients were 226 and 44 , respectively. ACPA values (mean $\pm \mathrm{SD}, \mathrm{U} / \mathrm{mL}$ ) were $280.3 \pm 376.8$ and 0.9 \pm 0.7 , and rheumatoid factor (RF) values were $174.8 \pm 302.6$ and $20.9 \pm$ 61.7 in the ACPA +ve and -ve groups, respectively. Mean ( $95 \%$ confidence interval) changes in SDAl at week 52 were $-11.3(-12.4$ to -10.3$)$ and $-8.0(-10.5$ to -5.5$)$, and those in J-HAQ were $-0.27(-0.34$ to -0.20$)$ and $-0.16(-0.34$ to 0.01$)$ in the ACPA +ve and -ve groups, respectively. In the Kaplan-Meier analysis, the retention rates of $A B A$ at week 52 in the ACPA +ve and -ve groups were $72.1 \%$ and $58.7 \%$, (discontinuation for any reason), and $91.6 \%$ and $75.7 \%$ (discontinuation because of lack of effectiveness), respectively. In a multiple regression analysis, the duration of disease ( $<1$ year) was associated with the change in SDAI at week 52 With respect to SDAl changes, the estimated difference of ACPA +ve and disease duration ( $<1$ year), ACPA +ve and disease duration ( $\geq 1$ year), and ACPA -ve and disease duration ( $<1$ year), versus ACPA -ve and disease duration ( $\geq 1$ year), were $-4.26(p=0.022),-0.82(p=0.618)$, and -0.93 $(p=0.716)$, respectively (Fig. 1). The estimated difference of ACPA +ve and $R F+v e, A C P A+v e$ and $R F$-ve, and ACPA -ve and RF + ve, versus ACPA -ve and RF -ve, were $-2.48(p=0.060),-2.77(p=0.107)$, and $-5.48(p=0.087)$, respectively.

Conclusion: A higher retention rate as well as better effectiveness of ABA on disease activity and physical function in ACPA +ve group versus ACPA -ve group were shown in the simple subgroup analysis. ABA effectiveness on the SDAI change was significantly better in patients with disease duration $<1$ year and ACPA +ve compared to those with ACPA -ve and disease duration $\geq 1$ year.

\section{REFERENCES:}

[1] Harrold LR et al. J Rheumatol 2018;45(1):32-39.

[2] Sokolove J et al. Ann Rheum Dis 2016;75(4):709-714.

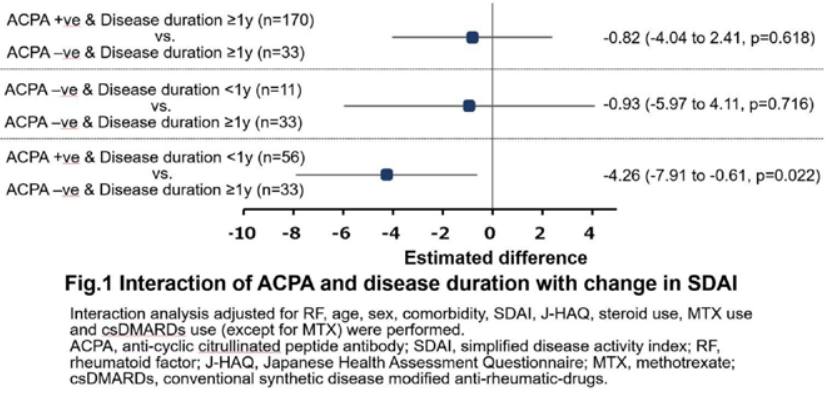

Disclosure of Interests: Kenta Misaki Speakers bureau: Eisai Co., Ltd., AbbVie GK, Eli Lilly Japan K.K., Ono Pharmaceutical Co., Ltd. Grant/research support from: Ono Pharmaceutical Co., Ltd., Eiichi Tanaka Speakers bureau: AbbVie GK, Asahi Kasei Pharma Corporation Astellas Pharma Inc, Ayumi Pharmaceutical Corporation, Chugai Pharmaceutical Co., Ltd., Eisai Co., Ltd., Eli Lilly Japan K.K., GlaxoSmithKline K.K., Kyowa Pharma Chemical Co., Ltd., Janssen Pharmaceutica K.K., Mochida Pharmaceutical Co., Ltd., Pfizer, Takeda Pharmaceutical Co., Ltd, and Teijin Pharma Ltd., Eisuke Inoue Speakers bureau: Pfizer Japan, Bristol-Myers Squibb K.K., Katsuki Tsuritani Employee of: Bristol-Myers Squibb K.K., Shigeru Matsumoto Employee of: Ono Pharmaceutical Co., Ltd., Hisashi Yamanaka Consultant of: Bristol-Myers Squibb K.K., masayoshi harigai Speakers bureau: AbbVie GK, Ayumi Pharmaceutical Corporation, Bristol-Myers Squibb K.K., Chugai Pharmaceutical Co., Ltd., Eisai Co., Ltd., Eli Lilly Japan K.K., Pfizer Japan Inc., and Takeda Pharmaceutical Co., Ltd., Consultant of: AbbVie GK Bristol-Myers Squibb K.K., Chugai Pharmaceutical Co., Ltd., Eli Lilly Japan K.K., and Gilead Sciences Inc., Grant/research support from: AbbVie GK, and Asahi Kasei Corp., Astellas Pharma Inc., Ayumi Pharmaceutical Corporation, Bristol-Myers Squibb K.K., Chugai Pharmaceutical Co., Ltd. Daiichi-Sankyo, Inc., Eisai Co., Ltd., Mitsubishi Tanabe Pharma Corporation., Nippon Kayaku Co., Ltd., Taisho Pharmaceutical Co., Ltd., and Takeda Pharmaceutical Co., Ltd. DOI: 10.1136/annrheumdis-2021-eular.1028 